S. E. Graversen

Nagoya Math. J.

Vol. 114 (1989), 123-133

\title{
A RIESZ DECOMPOSITION THEOREM
}

\author{
S. E. GRAVERSEN
}

\section{Introduction}

The topic of this note is the Riesz decomposition of excessive functions for a "nice" strong Markov process $X$. I.e. an excessive function is decomposed into a sum of a potential of a measure and a "harmonic" function. Originally such decompositions were studied by G.A. Hunt [8]. In [1] a Riesz decomposition is given assuming that the state space $E$ is locally compact with a countable base and $X$ is a transient standard process in strong duality with another standard process $\hat{X}$ having a strong Feller resolvent. Recently R.K. Getoor and J. Glover extended the theory to the case of transient Borel right processes in weak duality [6].

In a different direction K.L. Chung and M. Rao [2] discussed the Riesz representation and other related topics without assuming duality. Their conditions are analytic ones imposed on the potential density $u(x, y)$. To be precise, they assume that $u(x, y)$ is the potential density of a transient Hunt process and satisfies:

$u(x, y)$ is extended continuous in $y$ for any fixed $x, u(x, y)>0$ for any $(x, y)$ and $u(x, y)=\infty$ if and only if $x=y$.

It is proved in [2] that the Riesz decomposition holds for any excessive function. In [9] Ming Liao extends the results of Chung and Rao under slightly weaker assumptions.

The frame for this note is a transient Borel right process $X$ on a Lusin topological space $E$ with potential density $u(x, y)$ with respect to a given excessive reference measure $m$. No duality is assumed. In Section 1 -using pure potential theoretic standard $H$-cone technique-we construct the potential part $U_{\mu_{s}}$ of the Riesz decomposition of a given excessive function $s$. The assumption on $u(x, y)$ needed for this construction is properness and a point separating property of the dual operator $\hat{U}$ defined by 


$$
\hat{U} f(x):=\int f(y) u(y, x) m(d y),
$$

for non-negative Borel measurable functions $f$. In Section 2, which is more probabilistic in nature, it is proved under further conditions on $\hat{U}$ that the function $h$ in the decomposition $s=U \mu_{s}+h$ is harmonic in the following sense: $h=\tilde{P}_{K^{c}} h$ for every set $K$ compact in a specified topology $\tau$ where $\tilde{P}$. is the kernel associated with first penetration time. The main results are contained in Theorems 1.2 and 2.1, and the principal assumptions are contained in Assumptions 1.1 and 2.1.

\section{Section 0. Notation}

We shall use the standard notation ([1], [5]) of Markov processes without special mention. The following notation and minimal hypothesis will remain in force throughout.

(0.1) $E$ is a Lusin topological space ([5]) with Borel $\sigma$-field $\mathscr{B}(E)$.

(0.2) $\mathscr{B}(E)_{b}=\{f: E \rightarrow R \mid f$ bounded and Borel measurable $\}$ $\mathscr{B}(E)_{+}=\{f: E \rightarrow R \cup\{\infty\} \mid f$ non-negative and Bore] measurable $\}$ $\mathscr{B}(E)_{b+}=\mathscr{B}(E)_{b} \cap \mathscr{B}(E)_{+}$

(0.3) $\left(P_{t}\right)_{t \geqq 0}$ is a Borel semigroup on $(E, \mathscr{B}(E))$ with resolvent $\left(U^{\alpha}\right)_{\alpha \geqq 0}$.

(0.4) $X=\left(\Omega, \mathscr{F}, \mathscr{F}_{t}, X(t), \Theta_{t}, P^{x}\right)$ is a Borel right process in $E$ with transition function $\left(P_{t}\right)$, lifetime $\xi$, death point $\Delta$. (As usual $\Delta$ is adjointed to $E$ as an isolated point.)

(0.5) $m$ is a $\sigma$-finite excessive reference measure for $X$ :

(0.6) $q$ is an element of $\mathscr{B}(E)_{b_{+}}$so that $0<q \leqq 1$ and $0<U q \leqq 1$. $\left(U=U^{0}\right)$.

If $f, g$ are Borel measurable functions, $\langle f, g\rangle$ denotes the integral $\int f \cdot g d m$.

Using (0.5) and Theorem 14, Chapter IX in [4], we can construct a family of submarkovian Borel kernels $\left(\hat{P}_{t}\right)$ so that for $t>0$,

$$
\left\langle f, P_{t} g\right\rangle=\left\langle\hat{P}_{t} f, g\right\rangle \quad f, g \in \mathscr{B}(E)_{+} \cdot
$$

\section{Section 1.}

Let $\mathscr{E}$ denote the cone of $\left(P_{t}\right)$-excessive functions finite $[m]$ a.e., i.e. $s$ is in $\mathscr{E}$ if 
a) $s \in \mathscr{B}(E)_{+}$and $m((s=\infty))=0$

b) $P_{t} s(x) \leqq s(x), x \in E, t>0$

c) $\sup _{t>0} P_{t} s(x)=s(x), x \in E$.

The properties of the potential density required in this section are gathered in the following assumption.

Assumption 1.1. There exists a non-negative $\mathscr{B}(E) \times \mathscr{B}(E)$ measurable function $u(x, y)$ such that

$$
\begin{array}{ll}
U(x, d y)=u(x, y) m(d y) & \text { for } x \in E, \\
x \longrightarrow u(x, y) \text { is excessive for } X & \text { for } y \in E,
\end{array}
$$

and there exists $\left\{g, g_{i}, i \geqq 1\right\} \subseteq \mathscr{B}(E)_{b+}$ so that

1) $0<g, 0 \leqq g_{i} \leqq g$ and $\langle g, 1\rangle<\infty$.

2) $0<\hat{U}_{g}<\infty$ everywhere.

3) $\left(\hat{U}_{g_{i}} / \hat{U}_{g}, i \geqq 1\right)$ separates points in $E$.

4) If a Borel measurable function $k$ satisfies $\left\langle|k|, g_{i}\right\rangle<\infty$ and $\left\langle k, g_{i}\right\rangle \geqq 0$ for all $i$, then $k \geqq 0[m]$ a.e. and $m((k=\infty))=0$.

Denote by $Q_{+}$the positive rational numbers and let $A$ denote the smallest set of functions which is closed under finite sums, multiplication by positive constants, finite minimum and contains the following functions:

$$
\hat{U} g_{i}, \quad \hat{U} g, \quad \hat{U} \hat{P}_{t} g_{i} \wedge \hat{U} g_{i}, \quad \hat{U} \hat{P}_{t} g \wedge \hat{U} g \quad i \geqq 1, t \in Q_{+} .
$$

Lemma 1.1. For all $h$ in $A, h \cdot d m$ is an excessive measure for $X$ and there exists a constant $\alpha(h) \geqq 0$ so that $0 \leqq h \leqq \alpha(h) \hat{U} g$.

Proof. Since every $h$ in $A$ is finite everywhere, $h d m$ is $\sigma$-finite. Denote by $\tilde{A}$ the subset of $A$ having the desired properties. $\tilde{A}$ is then stable under finite sums, multiplication by positive constants and forming a finite minimum; therefore it suffices to prove that $\tilde{A}$ contains the set of functions listed above. Because $\hat{U} g \geqq \hat{U} g_{i}, i \geqq 1$, only the excessive property needs to be verified.

For $k$ in $\mathscr{B}(E)_{b_{+}}$and all $t$ in $Q_{+}$we have

$$
\left\langle k, \hat{U} \hat{P}_{t} g_{i}\right\rangle=\left\langle P_{t} U k, g_{i}\right\rangle \leqq\left\langle k, \hat{U} g_{i}\right\rangle,
$$

and likewise $\left\langle k, \hat{U} \hat{P}_{t} g\right\rangle \leqq\langle k, \hat{U} g\rangle$.

This means $\hat{U} \hat{P}_{t} g_{i} \leqq \hat{U} g_{i}$ and $\hat{U} \hat{P}_{t} g \leqq \hat{U} g[m]$ a.e. Thus we need only 
prove that $\hat{U} k d m$ is excessive for $k \in \mathscr{B}(E)_{b_{+}}$with $\hat{U} k<\infty$. But this is clear.

Lemma 1.2. For all $h$ in $A$ there is a unique $\sigma$-finite measure $\gamma_{h}$ on $(E, \mathscr{B}(E))$ so that

$$
h d m=\gamma_{h} U \quad\left(=\int \gamma_{h}(d y) U(y, d x)\right) .
$$

Proof. Let $h$ in $A$ be given. Define $\nu:=h d m$ and $\mu:=\alpha(h) g d m$. For all $k \in \mathscr{B}(E)_{b+}$ we have

$$
\begin{aligned}
\int k(y) \mu U(d y) & =\int U k(y) \mu(d y) \\
=\langle U k, \alpha(h) g\rangle & =\langle k, \alpha(h) \hat{U} g\rangle
\end{aligned}
$$

i.e. $\mu U=\alpha(h) \hat{U} g d m$, and thus $\nu \leqq \mu U$. The result is now implied by Theorem 4.2 and Proposition 1.1 in [7].

Remark. Easy computations show that $\gamma_{\hat{0} g}=g d m, \gamma_{\hat{\theta}_{i}}=g_{i} d m, \gamma_{h}=$ $\hat{P}_{t} g d m$ if $h=\hat{U} \hat{P}_{t} g \wedge \hat{U} g$ and $\gamma_{h}=\hat{P}_{t} g_{i} d m$ if $h=\hat{U} \hat{P}_{t} g_{i} \wedge \hat{U} g_{i}$.

Define $C:=\{s \in \mathscr{E} \mid\langle s, g\rangle\langle\infty\}$.

Lemma 1.3. $\int s(y) \gamma_{h}(d y)<\infty \quad$ for $s \in C, h \in A$.

Proof. Let $s \in C$ and $h \in A$ be given. Theorem IX T64 ([8]) implies that there exist $\left(\varnothing_{n}\right)_{n=1}^{\infty} \subseteq \mathscr{B}(E)_{b_{+}}$so that $U \varnothing_{n} \uparrow s$. Therefore

$$
\begin{aligned}
\int s(y) \gamma_{h}(d y) & =\sup _{n} \int U \varnothing_{n}(y) \gamma_{h}(d y)=\sup _{n}\left\langle\varnothing_{n}, h\right\rangle \\
& \leqq \sup _{n}\left\langle\varnothing_{n}, \alpha(h) \hat{U} g\right\rangle=\sup _{n}\left\langle U \varnothing_{n}, \alpha(h) g\right\rangle \leqq \alpha(h)\langle s, g\rangle\langle\infty .
\end{aligned}
$$

Define $\widetilde{\mathscr{H}}:=A-A$.

$\widetilde{\mathscr{H}}$ is a linear space of finite real valued Borel functions stable under finite minimum and finite maximum.

For $s$ in $C$, denote by $L(s, \cdot)$ the function on $\widetilde{\mathscr{H}}$ defined by

$$
L(s, h):=\int s d r_{h_{1}}-s d r_{h_{2}},
$$

where $h_{i} \in A, i=1,2$ and $h=h_{1}-h_{2}$.

The proof of Lemma 1.3 shows that $L(s \cdot)$ is a well defined positive linear functional on $\widetilde{\mathscr{H}}$ and that $s \rightarrow L(s, h)$ is additive for each $h$ in $\widetilde{\mathscr{H}}$. Likewise, if $s, s_{1}$ and $s_{2}$ are in $C$ with $s=s_{1}+s_{2}$, then we have 


$$
L(s, h) \geqq L\left(s_{1}, h\right) \quad \text { for } h \in \widetilde{\mathscr{H}}_{+} .
$$

Define $\mathscr{H}:=\{h / \hat{U} g \mid h \in \widetilde{\mathscr{H}}\}$.

Then $\mathscr{H}$ is a linear space of bounded real valued Borel functions stable under finite infimum and finite maximum, containing constants and separating points in $E$. Furthermore, $\mathscr{H}_{+}$is separable in the supnorm. Let $\bar{E}$ denote the compactification of $E$ by means of $\mathscr{H}_{+}$([5]). $\bar{E}$ is a compact metric space with Borel field $\mathscr{B}(\bar{E})$. The assumptions imply that $E \in \mathscr{B}(\bar{E})$ and $\mathscr{B}(E)=\mathscr{B}(\bar{E}) \cap E$. Since $E$ is dense in $\bar{E}$, every element $h$ in $\mathscr{H}$ has a unique continuous extension $\bar{h}$ to $\bar{E}$, and $\overline{\mathscr{H}}:=$ $\{\bar{h} \mid h \in \mathscr{H}\}$ is dense in $\mathscr{C}(\bar{E})$.

Daniell's theorem implies that every positive linear functional $\bar{L}$ on $\overline{\mathscr{H}}$ is of the form

$$
\bar{L}(\bar{h})=\int_{E} \bar{h} d \mu_{\bar{L}}+\int_{\bar{E} \backslash E} \bar{h} d \bar{\mu}_{\bar{L}}
$$

where $\mu_{\bar{L}}$ and $\bar{\mu}_{\bar{L}}$ are uniquely determined bounded measures on $(\bar{E}, \mathscr{B}(\bar{E}))$ satisfying $\bar{\mu}_{\bar{L}}(E)=0$ and $\mu_{\bar{L}}(\bar{E} \backslash E)=0$. Thus $\mu_{\bar{L}}$ is a bounded measure on $(E, \mathscr{B}(E))$.

Every positive linear functional $L$ on $\widetilde{\mathscr{H}}$ can be regarded as a positive linear functional $\bar{L}$ on $\overline{\mathscr{H}}$ through the formula

$$
\bar{L}(\bar{h})=L(\tilde{h}) \quad \text { where } \bar{h}=\overline{\bar{h} / \hat{U} g} .
$$

Since $\hat{U} g>0$ everywhere, this proves that every positive linear functional $L$ on $\widetilde{\mathscr{H}}$ is of the form

$$
L(h)=\int_{E} h d \mu_{L}+\int_{\bar{E} \backslash E} \overline{h / \hat{U}_{g}} d \bar{\mu}_{L}
$$

where $\mu_{L}$ is a uniquely determined $\sigma$-finite measure on $(E, \mathscr{B}(E))$ and $\bar{\mu}_{L}$ is a bouned measure on $(\bar{E}, \mathscr{B}(\bar{E}))$ so that $\bar{\mu}_{L}(E)=0$.

Notation. A positive linear functional $L$ on $\widetilde{\mathscr{P}}$ is called singular if $\mu_{L}=0$, and it is called an integral if $\bar{\mu}_{L}=0$.

Remark. Daniell's theorem implies that a positive linear functional $L$ on $\widetilde{\mathscr{H}}$ is an integral if and only if

$$
L\left(f_{n}\right) \downarrow 0 \quad \text { if } \quad\left(f_{n}\right) \subseteq \widetilde{\mathscr{H}}_{+} \quad \text { and } \quad f_{n} \downarrow 0 .
$$


A consequence of this is that $L$ is an integral if there exists $\left(L_{L}\right)$ all integrals, so that $L_{n}(h) \uparrow L(h)$ for all $h$ in $\widetilde{\mathscr{H}}_{+}$.

The proof of the following Lemma is immediate and therefore omitted.

LEMMA 1.4. Let $L_{1}$ and $L_{2}$ be two positive linear functionals on $\widetilde{\mathscr{H}}$ so that $L_{1}(h) \geqq L_{2}(h)$ for all $h$ in $\widetilde{\mathscr{H}}_{+}$. Then

$L_{1}$ is an integral (singular) $\Longrightarrow L_{2}$ is an integral (singular).

Translating these results gives that for $s$ in $C$

$$
L(s, h)=\int_{E} h d \mu_{s}+\int_{\bar{E} \backslash E} \overline{\bar{h} / \hat{U} g} d \bar{\mu}_{s}, \quad h \in \widetilde{\mathscr{H}},
$$

where $\mu_{s}$ is a uniquely determined $\sigma$-finite measure on $(E, \mathscr{B}(E))$ and $\bar{\mu}_{s}$ is a bounded measure on $\left(\bar{E}, \mathscr{B}(\bar{E})\right.$ so that $\bar{\mu}_{s}(E)=0$. Furthermore $s \in C$ is called singular (an integral) if $L(s, \cdot)$ is singular (an integral).

Denote by $\boldsymbol{S}(\boldsymbol{I})$ the set of singular (integral) elements in $C$.

Theorem 1.1. Let $s_{1}$ and $s_{2}$ in $C$ be given. Assume $s_{1} \gg s_{2}$ (strong order). Then

$$
s_{1} \in S(I) \Longrightarrow s_{2} \in S(I) .
$$

Proof. Use Lemma 1.4 and formula (1.2).

In order to state the main theorem of this section, we need to define the so-called "harmonic" elements in $C$.

Definition. $s$ in $C$ is called "harmonic" iff $u \in I$ and $s \gg u \Rightarrow u \equiv 0$. Let $\boldsymbol{H}$ denote the set of "harmonic" elements in $\boldsymbol{C}$. Clearly $\boldsymbol{S} \subseteq \boldsymbol{H}$.

Theorem 1.2. For $s$ in $C$, the following decomposition is valid,

$$
s=U \mu_{s}+s_{1},
$$

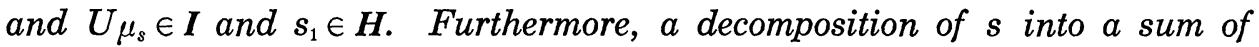
an integral and a "harmonic" element is unique.

The proof of Theorem 1.2 is based on the following lemmas.

LEMma 1.5. $s$ in $C$ admits at most one decomposition of the form $u+v$, where $u \in \boldsymbol{I}$ and $v \in \boldsymbol{H}$.

Proof. Lemma 1.5 is related to the "Riesz splitting proparty" see 
[12]. Let $s$ in $C$ be given and assume $s=u_{i}+v_{i}$, where $u_{i} \in \boldsymbol{I}$ and $v_{i} \in \boldsymbol{H}$ for $i=1,2$.

$$
u_{2} \ll s \Longrightarrow u_{2}=u_{12}+u_{22}, \quad \text { where } u_{12} \ll u_{1} \text { og } u_{22} \ll v_{1} \text {. }
$$

Since $u_{22} \ll u_{2}$ and $u_{22} \ll v_{1}, u_{22}$ equals 0 by Theorem 1.1. Thus $u_{2} \ll u_{1}$ which by symmetry gives the result.

Lemma 1.6. $s=U \mu_{s}$ for all $s$ in $I$.

Proof. Let $s$ in $C$ be given. For all $i \geqq 1$ we have

$$
\begin{aligned}
\left\langle U \mu_{s}, g_{i}\right\rangle & =\int \hat{U} g_{i} d \mu_{s}=L\left(s, \hat{U} g_{i}\right) \\
& =\int s d r_{\hat{U} g_{i}}=\left\langle s, g_{i}\right\rangle<\infty,
\end{aligned}
$$

which by Assumption 1.1 provides the result.

Lemma 1.7. For $s$ in $C, A_{s}=\{u \in C \mid s \gg u, u \in I\}$ admits an upper bounded in the strong order.

Proof. Let $s$ in $C$ be given. Since $I$ is stable under addition, it follows that $A_{s}$ is upwards filtering in the strong order. Theorem 1.5, p. 198 [1] now implies the existence of a sequence $\left(u_{n}\right) \subseteq A_{s}$ so that $u_{n} \ll u_{n+1}$, $n \geqq 1$ and $\sup _{n} u_{n}=\sup \left\{u \mid u \in A_{s}\right\}$ (pointwise). $u_{\infty}:=\sup _{n} u_{n}$. A simple argument shows that $u_{\infty} \in C$ and $u_{\infty} \ll s$.

Because $u_{n} \ll u_{n+1}, n \geqq 1$, there exists $\left(\varnothing_{k}^{n}\right)_{k, n \geqq 1} \subseteq \mathscr{B}(E)_{b+}$ so that

a) $\varnothing_{k}^{n} \leqq \varnothing_{k}^{n+1}, n \geqq 1, k \geqq 1$,

b) $U\left(\varnothing_{k}^{n}\right) \uparrow_{k \rightarrow \infty} u_{n}, n \geqq 1$.

a) and b) imply that $U\left(\varnothing_{n}^{n}\right) \uparrow_{n \rightarrow \infty} u$. Therefore

$$
L(u, h)=\lim _{n}\left\langle\varnothing_{n}^{n}, h\right\rangle \geqq \lim _{n}\left\langle\varnothing_{n}^{n_{0}}, h\right\rangle=L\left(u_{n_{0}}, h\right)
$$

for all $h$ in $\widetilde{\mathscr{H}}$ and all $n_{0} \geqq 1$.

But by monotone convergence we have

$$
\int u_{n} d r_{h} \underset{n \rightarrow \infty}{\longrightarrow} \int u_{\infty} d r_{h} \quad \text { for all } h \text { in } \widetilde{\mathscr{H}},
$$

and thus $L\left(u_{n}, h\right) \uparrow_{n \rightarrow \infty} L\left(u_{\infty}, h\right)$ for $h \in \widetilde{\mathscr{H}}_{+} \cdot u_{\infty}$ is therefore an integral. The fact that $u_{\infty}$ is an upper bound for $A_{s}$ is immediate.

Proof of Theorem 1.2. Only the existence of a decomposition remains to be shown. Let $s$ in $C$ be given and let $u$ be the upper bound for $A_{s}$ 
constructed in Lemma 1.7,

$$
u \ll s \Longrightarrow \exists v \in C: s=u+v \text {. }
$$

If $v$ does not belong to $\boldsymbol{H}$, there exists $v_{1} \in \boldsymbol{I} \backslash\{0\}$ so that $v \gg v_{1}$, but this leads to a contradiction because in that case $u+v_{1}$ will be an element of $A_{s}$. The proof of Theorem 1.2 is thus complete.

\section{Section 2.}

Throughout the rest of this paper we will assume, together with Assumption 1.1, the following

Assumption 2.1. There exists a second countable metric topology $\tau$ on $E$ so that

a) $\mathscr{B}(\tau)=\mathscr{B}(E)$.

b) Every function in $\widetilde{\mathscr{H}}$ is $\tau$-continuous.

c) $\tau$ is finer than the given topology on $E$.

d) There exists a Borel semipolar set $B$ so that $\left.\tau\right|_{B c}$ is coarser than the fine topology restricted to $B^{c}$.

The aim of this section is to show that the elements in $\boldsymbol{H}$ deserve the name "harmonic" elements.

Definition. For every $F \in \mathscr{B}(E)$, let $\tilde{T}_{F}$ denote the penetration time into $F$, i.e.

$$
\tilde{T}_{F}(\omega):=\inf \left\{t \geqq 0 \mid[0, t] \cap\left\{u \geqq 0 \mid X_{u}(\omega) \in F\right\} \text { is uncountable }\right\}
$$

$\tilde{T}_{F}$ is a stopping time and exact terminal time satisfying $\tilde{T}_{F} \cdot \Theta_{\tilde{T}_{F}}=0$ on $\left\{\tilde{T}_{F}<\infty\right\}$ [3]. For all $s$ in $\mathscr{E}$ and $F$ in $\mathscr{B}(E)$, denote by $\tilde{P}_{F} s(x)$ the value $E_{x}\left(s\left(X_{\tilde{T}_{F}}\right)\right)$. The properties of $\tilde{T}_{F}$ imply that $x \rightarrow \tilde{P}_{F} s(x)$ is again an element of $\mathscr{E}$.

Lemma 2.1. Let $O$ denote a $\tau$-open set. There exists a finely open set $V$ so that $O \cap B^{c}=V \cap B^{c}$ and

$$
\tilde{P}_{o} s(x)=P_{V} s(x)=E_{x}\left(s\left(X\left(T_{V}\right)\right)\right)
$$

for $x \in E$ and $s \in \mathscr{E}$, where $T_{V}$ is the first hitting time to $V$.

Proof. Let $s \in \mathscr{E}$ be given. Using Assumption $2.1 \mathrm{~d}$ ), there exists a finely open set $V$ so that $O \cap B^{c}=V \cap B^{c}$. Since $B$ is semipolar and thus only visited countably often, we have $\tilde{T}_{o}=\tilde{T}_{o \cap B^{c}}$ and $\tilde{T}_{V}=\tilde{T}_{V \cap B^{c}}$ a.s. 
$P^{x}$ for $x \in E$. But since $V$ is a finely open set, $T_{V}=\tilde{T}_{V}$ a.s. $P^{x}$ for $x \in E$. Thus

$$
\tilde{T}_{o}=T_{V} \quad \text { a.s. } \text { for } x \in E
$$

so that $\tilde{P}_{o} s=P_{V} s$.

LemMA 2.2. Let $s \in \boldsymbol{H}$ and $K$ a $\tau$-compact set be given. There exists $a \tau$-open set $O$ containing $K$ so that $s \neq \tilde{P}_{o} s$.

Proof. Since $s \notin \boldsymbol{I}$, there exists $h,\left\{h_{n}\right\}_{n}$ in $\widetilde{\mathscr{H}}_{+}$, so that $h_{n} \uparrow h$ and $L(s, h)-\sup _{n} L\left(s, h_{n}\right)=\beta>0$. $\hat{U} g$ is strictly positive, so we can choose $\varepsilon>0$ so that

$$
K \cong\{\hat{U} g>\varepsilon\} .
$$

Pick an $\alpha>0$ so that $\alpha \varepsilon^{-1}\langle s, g\rangle\left\langle\beta\right.$. Since $h-h_{n} \downarrow 0$, a Dini argument implies the existence of a $\tau$-open set $O$ and an $n_{0} \geqq 1$, so that

$$
K \subseteq O \subseteq\{\hat{U} g>\varepsilon\} \quad \text { and } \quad O \subseteq\left\{h-h_{n_{0}}<\alpha\right\} .
$$

We claim that $s \neq \tilde{P}_{0} s$. Assume the opposite. Choose $V$ finely open according to Lemma 2.1 and the given set $O$. Since $V$ is finely open and $B$ is semipolar, we can find, since $s=\tilde{P}_{o} s=P_{v} s$ (see page 88 in [1]), $\left(\varnothing_{k}\right)_{k} \subseteq \mathscr{B}(E)_{b+}$ satisfying $U \varnothing_{k} \uparrow s$ and $\operatorname{supp}\left(\varnothing_{k}\right) \subseteq V \cap B^{c} \subseteq O$ for $k \geqq 1$. Now

$$
\begin{aligned}
L(s, h)-L\left(s, h_{n_{0}}\right) & =\lim _{h \rightarrow \infty}\left\langle\varnothing_{k}, h-h_{n_{0}}\right\rangle \leqq \alpha \lim _{k} \sup \left\langle\varnothing_{k}, 1\right\rangle \\
& \leqq \alpha \varepsilon^{-1} \lim _{k} \sup \left\langle\varnothing_{k}, \hat{U} g\right\rangle \leqq \alpha \varepsilon^{-1}\langle s, g\rangle<\beta
\end{aligned}
$$

i.e. we have derived a contradiction.

Remark. A similar argument implies that if $s$ in $C$ satisfies $s=\tilde{P}_{o} s$ for a $\tau$-relative compact $\tau$-open set $O$, then $s \in I$.

Definition. $s$ in $\mathscr{E}$ is said to be minimal if, whenever $u, v$ are in $\mathscr{E}$ and $s=u+v$, both $u$ and $v$ are proportional to $s$.

Notation. The set of minimal elements of $C, \boldsymbol{I}$ and $\boldsymbol{H}$ will be denoted $C^{e}, \boldsymbol{I}^{e}$ and $\boldsymbol{H}^{e}$.

It is immediately seen that we have the following set identity

$$
C^{e}=\boldsymbol{I}^{e} \cup \boldsymbol{H}^{e} \text {. }
$$

Lemma 2.3. For $s$ in $\boldsymbol{H}^{e}$ and every $\tau$-compact set $K$ we have $s=\tilde{P}_{K^{e}} s$. 
Proof. Let $s$ in $H^{e}$ and $K$ a $\tau$-compact set be given. According to Lemma 2.2 there exists a $\tau$-open set $O$ containing $K$ so that $s \neq \tilde{P}_{o} s$. Choose $V$ finely open according to Lemma 2.1 and the given $O$,

$$
s=P_{V} s+a
$$

where

$$
a= \begin{cases}0 & \text { on the fine closure of } V \\ s-P_{V} s & \text { elsewhere }\end{cases}
$$

Using a theorem of G. Mokobodzki ([12]), we have $s=v+\underline{R} a$, where $v \in \mathscr{E}$ and $v \leqq P_{V} s$ and $\underline{R} a$ is the excessive regularisation of

$$
R a=\inf \{u \mid u \geqq a \text { and } u \text { supermedian }\} .
$$

Since $s$ is minimal and $a \neq 0$, we have $s=\beta \underline{R} a$ for some $\beta$ in $(0,1]$. Let $O_{1}$ denote a $\tau$-open set so that $K \subseteq O_{1} \subseteq \bar{O}_{1}^{\tau} \subseteq O$, where $-\tau$ denotes the $\tau$-closure. The existence of $O_{1}$ is ensured by the regularity of $\tau$. We now now claim that $s=\tilde{P}_{0_{2}} s$, where $O_{2}=\left(\bar{O}_{1}^{\mp}\right)^{c}$. Denote by $V_{2}$ the finely open set chosen according to Lemma 2.1 and $O_{2} . s=P_{V_{2}}$ on $V_{2}$, and since we have $V_{2} \cap B^{c}=O_{2} \cap B^{c} \supseteqq O^{c} \cap B^{c}=V^{c} \cap B^{c} \supseteqq\left(\bar{V}^{f}\right)^{c} \cap B^{c}, \quad s=P_{V_{2}} s \quad$ on $\left(\bar{V}^{f}\right)^{c} \backslash B$. But $B$ is semipolar and $\left(\bar{V}^{f}\right)^{c}$ finely open, so this implies $s=P_{V_{2}} s$ on $\left(\bar{V}^{f}\right)^{c}$ and thus $\beta^{-1} P_{V_{2}} \geqq \underline{R}\left(s-P_{V} s\right)$. The conclusion $s=P_{V_{2}} s$ is now immediate, and since $K^{c} \supseteqq O_{2}$, we also have $s=\tilde{P}_{K c} s$.

Theorem 2.1. For $s$ in $H$ and every $\tau$-compact set $K$ we have $s=\tilde{P}_{K} s$.

Proof. Let $s$ in $\boldsymbol{H}$ be given. A famous theorem of G. Mokobodzki ([13], see also [14]) ensures that $s$ can be represented as an integral of elements belonging to $C^{e}$. But this implies, together with Theorem 1.1 and formula (2.1) that $s$ can be represented as an integral of excessive functions belonging to $\boldsymbol{H}^{e}$. The conclusion of the theorem is now a consequence of Lemma 2.3 and Fubini's theorem.

An example which fits into the framework of this paper but not into that of [6]:

$X$ is a Brownian motion of $(-1,1)$ which is reflected at 0 when approaching from the right. Here no dual process exists, but the assumptions of this paper is fulfilled with $\tau$ equal to the sum topology on $(-1,0) \cup[0,1]$. 


\section{REFERENCES}

[1] Blumenthal, R. M., Getoor, R. K., Markov processes and potential theory, New York, Academic Press, 1968.

[2] Chung, K. L., Rao, M., A new setting for potential theory, Ann. Inst. Fourier, 30 (1980), 167-198.

[ 3 ] Dellacherie, C., Capacités et processus stochastiques, Berlin-Heidelberg-New York, Springer Verlag, 1972.

[4 ] Dellacherie, C., Meyer, P. A., Probabilités et potentiel: Théorie discrète du potentiel, Herman, 1983.

[5] Getoor, R. K., Ray processes and right processes, Lecture Notes in Math., 440, Berlin-Heidelberg-New York, Springer Verlag, 1975.

[ 6 ] Getoor, R. K., Glover, J., Riesz decompositions in Markov process theory, Trans. Amer. Math. Soc., 285 (1984), 107-132.

[ 7 ] — Markov processes with identical excessive measures, Math. Z., 184 (1983), $187-300$.

[ 8 ] Hunt, G. A., Markov processes and potentials I, Illinois J. Math., 1 (1957), 44-93.

[9] Liao, M., Riesz representation and duality of Markov processes, Séminaire de Probabilités XIX, 366-396, Lecture Notes in Math., 1123.

[10] Meyer, P. A., Probability and potentials, Blaisdell, 1966.

[11] —- Processus de Markov: la frontière de Martin, Lecture Notes in Math., 77, Berlin-Heidelberg-New York, Springer Verlag, 1968.

[12] Mokobodzki, G., Densité relative de deux potentiels comparables, Lecture Notes in Math., 124, Sem. de Prob. IV (1970), 170-194.

[13] —-, Dualité formelle et représentation des fonctions excessives, Actes du Congrès Int. des Mathematiciens, t. 2, 1970, 531-535.

[14] Walsh, J. B., The cofine topology revisited, Proc. Symp. in Pure Math., 31 (1977), 131-152. AMS, Providence.

Department of Mathematics

Aarhus University

Ny Munkegade, 8000 Aarhus $C$

Denmark 
